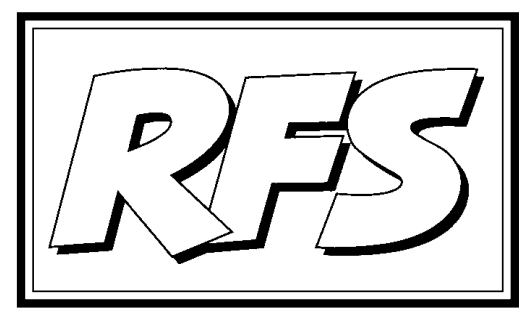

Revista de Fomento Social, 55 (2000), 391-418

\title{
Percepción y tipología del trabajo de los menores: un acercamiento cualitativo al caso de Andalucía ${ }^{1}$
}

Rafael SERRANO DEL ROSAL*

\section{INTRODUCCIÓN}

El trabajo y la explotación laboral infantil es uno de los problemas sociales que, actualmente, generan más indignación y sentimientos de repulsa en las sociedades desarrolladas. De hecho, la estimación realizada por la OIT y la UNICEF sobre el número de niños trabajadores en el mundo

1 Este artículo tiene como base empírica una investigación más amplia titulada El trabajo de los menores en Andalucía, financiada por la Dirección General de Atención al Niño, Consejería de Asuntos Sociales, J unta de Andalucía, en el marco de un convenio de colaboración entre ésta y las organizaciones sindicales UGT y CCOO, y realizada por el Instituto de Estudios Sociales Avanzados de Andalucía/CSIC bajo la dirección del autor de este artículo. A estas instituciones y a las personas que han colaborado en la ejecución de este trabajo, especialmente a J esús Ligero, muchas gracias.

* Doctor en Ciencias Políticas y Sociología por la Universidad de Granada. Es investigador del IESA de Andalucía. 
actual - 250 millones- ha sido uno de los datos más reproducidos, tanto por la literatura especializada, como por la prensa y los medios de comunicación en general. Ello, sin embargo, no significa que haya un conocimiento exhaustivo sobre la naturaleza del problema, ni tan siquiera que exista un lugar común sobre lo que es o significa el trabajo infantil, lo cual dificulta la identificación de los condicionantes y los efectos de este fenómeno social. De hecho, si se hace el esfuerzo analítico de describir los distintos condicionantes que generalmente se citan como explicativos del trabajo infantil, así como sus consecuencias, se puede observar que no sólo no son todos complementarios sino que, por el contrario, muchos son completamente opuestos, lo cual, posiblemente, sea un síntoma de que los análisis realizados no parten de los mismos conceptos o no se refieren al mismo tipo de trabajo infantil (ver cuadro 1).

CUADRO 1

\section{Enfoques sobre los condicionantes del trabajo infantil}

\section{CONSECUENCIAS DEL TRABAJ O INFANTIL}

- Mecanismo de aprendizaje y socialización

- Factores demográficos: tamaños de la familia, número de personas dependientes etc.

- Desestructuración familiar

- Explotación económica

- Subdesarrollo y estructura del mercado de trabajo

- Estrategias de supervivencia

- Racionalidad familiar

- Inadecuación del sistema escolar

- Valoraciones sociales: necesidades sociales, necesidad de pertenencia, consumo etc.

- Efectos físicos

- Efectos psicológicos

- Efectos sobre la escolaridad

- Efectos sobre la pobreza

- Efectos sobre la integración social

Fuente: Rojas FLoRES, J., «El trabajo infantil en Chile: algunas ideas para el debate», en Fernández, M. (Ed.) Economía y trabajo en Chile 1997-1998, PET 1998. 
En España, pese a la importancia que se le da al fenómeno y a la sensibilidad expresada desde muchos sectores de la sociedad, se sigue careciendo de un estudio fiable sobre su magnitud real (UGT, 1998: 3). No obstante, a lo largo de los noventa, diferentes instituciones han producido una serie de datos sobre la incidencia del fenómeno en España. Éstas dan cifras que oscilan entre los 250.000 y los 800.000 niños trabajadores. Las diferencias entre unas cifras y otras ponen demanifiesto al menos dos hechos que se retroalimentan repercutiendo en la fiabilidad de los datos aportados. Por un lado, la falta de una definición común sobre el tipo o tipos de trabajo infantil que se están considerando en la medición. Y, por otro, la carencia de indicadores directos para medir su incidencia real.

Si para el conjunto de España los estudios sobre el trabajo infantil son escasos y poco fiables, para el caso concreto de la Comunidad Autónoma Andaluza éstos son inexistentes. Lo único encontrado son referencias a su casuística con poco o ningún fundamento empírico. Es decir, suposiciones más o menos informadas sobre las características de este fenómeno en Andalucía, especialmente en su relación con algunos sectores de la economía rural como, por ejemplo, los jornaleros.

A nuestro juicio, todo ello fundamenta la necesidad de que cualquier trabajo que pretenda profundizar con cierto rigor sobre el fenómeno deba comenzar por fundamentar y comprender la percepción social y la conceptuación que de él se hace, como paso previo, tanto para analizar sus causas y sus consecuencias, como para abordar su medición o la elaboración de cualquier tipo de política pública. La ausencia de dicha labor de comprensión provoca que se consideren como realidades de hecho, tópicos o situaciones que forman parte del imaginario colectivo de una sociedad.

Todo ello justifica que el presente artículo tenga los siguientes objetivos: primero, analizar los diferentes conceptos que socialmente se manejan en Andalucía sobre el trabajo infanto-juvenil ${ }^{2}$ (TIJ ), así como la valoración que sobre éste hacen los diferentes agentes sociales. Y, segundo, construir tipos

2 En este artículo se va a utilizar el término trabajo infanto-juvenil (TIJ) en lugar del de trabajo infantil con objeto de dar mejor cobertura conceptual a la investigación realizada. Puesto que al hablar de el trabajo de los menores, nos referimos a menores de edad laboral que son, en la mayoría de los casos, personas menores de 16 años, aunque también en algunos casos, pudiera referirse a menores de 18 años. La población de referencia de está investigación la forman un conjunto de andaluces que por su edad podríamos encuadrar tanto en la infancia, como en la adolescencia o primera etapa de la juventud. Por tanto, el concepto TIJ se adapta mejor a la población objeto de estudio que el de trabajo infantil. 
ideales de TIJ con los que poder analizar las características, condiciones, causas y consecuencias de este fenómeno en sus diferentes manifestaciones.

\section{METODOLOGÍA}

Para implementar los objetivos arriba expuestos, se diseñó y ejecutó una investigación de carácter cualitativo. Con la utilización de técnicas cualitativas se ha tratado de conocer en profundidad el origen, características y evolución del fenómeno estudiado, atendiendo antes que a la medición, a la compresión y conceptuación que hacen del mismo los diferentes agentes sociales directa o indirectamente implicados.

Concretamente, la técnica de investigación empleada ha sido la entrevista semiestructurada. Ésta permite recabar la opinión y percepción que tienen los informantes clave sobre los distintos aspectos del fenómeno objeto de estudio al tiempo que posibilita que éstos traten y desarrollen otros aspectos del problema que no se conocían o no se habían tenido en cuenta con anterio ridad. Concretamente, en esta investigación se construyó una guía de entrevista con una serie de preguntas abiertas que el entrevistador trataba de que contestasen todos los informantes, siguiendo el modelo de una conversación entre iguales y no de un intercambio formal de preguntas y respuestas (Taylor, S. y Bogdan, R. 1994). De esta forma se consiguió obtener información sobre un mismo conjunto de aspectos del fenómeno estudiado por parte de todos los informantes y, a la vez, que el instrumento no se convirtieseen un elemento queconstriñese las posibilidades informativas de los entrevistados, tanto en el enfoque que estos pudieran dar a los temas que se les proponían, como en los nuevos temas que éstos pudieran abordar o poner en relación con el anterior.

Las entrevistas, 86 en total, se realizaron en todas las provincias de la comunidad autónoma andaluza durante 1999. En cada una de las provincias se realizaron entrevistas en distintos núcleos poblacionales. El objetivo era contar con informantes, tanto en las capitales de provincia, como en otras comarcas o pueblos que, por sus características especiales, se considerara que el fenómeno estudiado podía tener especial incidencia o características diferenciales.

La selección de los informantes clave se realizó de dos formas diferentes. En primer lugar se seleccionaron informantes en instituciones u organizaciones que por la actividad que desarrollan podían tener contacto con el

\section{RFS}


fenómeno estudiado (sindicatos, servicios sociales municipales, delegaciones de educación, directores de colegios e I.E.S, inspección de trabajo, etc.). En segundo lugar se seleccionaron informantes de asociaciones u organizaciones no gubernamentales que, igualmente, podían conocer directa o indirectamente el fenómeno estudiado. Para ello se utilizó la técnica conocida como muestreo bola de nieve ${ }^{3}$.

La información aportada por lo informantes se transcribió y analizó ordenando los resultados obtenidos en los dos apartados siguientes, cada uno de los cuales responde a uno de los objetivos que se han planteado en este artículo. En el primero se analiza la percepción y conceptuación social del fenómeno del TIJ en Andalucía. Y, en el segundo, se construyen tres tipos ideales de TIJ con objeto de profundizar en el análisis de las diferentes causas y consecuencias de este complejo fenómeno social.

\section{PERCEPCIÓN SOCIAL Y CONCEPTUACIÓN DEL TRABAJ O INFANTO- J UVENIL EN ANDALUCÍA}

El objetivo deeste apartado es identificar y profundizar en el conocimiento de los conceptos que sobre el TIJ manejan los distintos agentes sociales andaluces que de forma directa $o$ indirecta tienen contacto con esta realidad. Ello es importante, tanto para comprender como se entiende el TIJ en la sociedad andaluza, como para interpretar las acciones u omisiones habidas en relación al mismo.

Lo primero destacable en este sentido es quela mayoría de los informantes abordaban el fenómeno de estudio en términos de desviación social que debe de ser extirpada. Situación que, generalmente, se iba transformando, explícita o implícitamente, conforme cada uno de los informantes trataba de definir qué consideraba como TIJ o dónde pondría la frontera entre el que debe ser considerado como sinónimo de explotación, y el que no tiene por qué serlo, es decir, entre "child labour" y "child work"4

3 Esta técnica consiste en conocer a algunos informantes y lograr que estos nos presenten a otros. TAYLOR, S. y BOGDAN, R. (1994).

4 La complejidad e importancia de los debates conceptuales sobre lo que debe considerarse trabajo infantil es tal que incluso organizaciones de carácter internacional como la OIT y UNICEF, que se caracterizan por tener como meta abolir completamente el trabajo infantil, hoy en día se han visto abocadas a reconocer que no toda forma de trabajo daña a los niños. Esta distinción entre el trabajo en un sentido amplio y no necesariamente transgresor, y el 
Dicho comportamiento refleja, al menos, dos realidades. En primer lugar, que gran parte de los informantes no habían reflexionado con profundidad sobre este fenómeno hasta el momento de la entrevista, mucho menos centrándolo en su entorno social más cercano, lo que explica la ambigüedad en sus razonamientos. En segundo lugar, que frente a este fenómeno generalmente se aplica una doble moral, por un lado, una socialmente correcta y ampliamente compartida que se plasma de forma casi automática cuando se aborda el fenómeno en términos generales y, otra, más heterogénea y comprensiva con el TIJ que se utiliza para interpretarlo en el entorno social próximo.

Por tanto, aunque a priori la conceptualización que hacen los diferentes agentes sociales del TIJ es muy parecida, posteriormente, cuando se profundiza en su discurso, se encuentran diferencias que parecen tener como base dos factores. Estos son: el tipo de relación que mantienen con el fenómeno los informantes seleccionados y la cercanía o conocimiento directo que tienen de él.

Los entrevistados que pertenecen al sistema educativo, por ejemplo, suelen conceptuar el TIJ en relación con el absentismo y la no escolarización, ya sean éstos la causa o la consecuencia de aquél. De hecho, su actitud de rechazo inicial ante el TIJ parece relajarse cuando se les pide que valoren situaciones en las que el trabajo no implica necesariamente un abandono de las aulas -fines de semana o vacaciones-. Algunos incluso señalan que este tipo de trabajo puede ser una actividad positiva para la socialización del menor.

Tal discurso es algo diferente cuando se trata de educadores que conocen o realizan su labor en centros educativos inmersos en barriadas marginales. Estos abordan el fenómeno también en su relación con la educación pero planteando problemáticas concretas que la trascienden. Por un lado, abundan en los problemas que se les plantean para conseguir que los menores estén escolarizados hasta los 16 años en un ámbito cultural y social en el que la educación no es un valor. Por otro, resaltan las dificultades que encuentran para hacer una adaptación curricular que interese e incentive a los menores y que convenza, a la vez, a sus familias de la importancia de la escolarización.

trabajo en el sentido de explotación, la asumen acuñando dos conceptos claves para el debate internacional sobre el trabajo infantil: child work para el primer tipo y child labour para el segundo. 
Este último tipo de educadores no considera el trabajo como la causa principal del absentismo o la no escolarización de estos menores, sino que se trataría de la consecuencia menos grave. Las principales causas serían dos que, además, están íntimamente relacionadas. Por un lado, la exclusión social y la pobreza, aunque ponen especial énfasis en la primera: generalmente se refieren a la desestructuración familiar, el abandono, los problemas de drogadicción, etc. Por otro, las diferentes pautas culturales y escalas de valores que se tienen en éstos ámbitos sociales. Es decir, los diferentes conceptos de cuando un niño pasa a ser adulto, sobre la importancia de la educación, o sobre otros factores que, por lo general, se encuadran en lo que se ha venido a llamar cultura de la marginación.

Si se centra la atención en el discurso de aquellos entrevistados que trabajan en el ámbito de los servicios sociales, se encuentra que suelen abordar el fenómeno del TIJ desde una doble perspectiva. En primer lugar, están aquellos que sólo contemplan en su discurso aquel tipo de TIJ que está relacionado con la exclusión social, lo cual tiene como efecto que cuando el TIJ se da en un ámbito en el que más que una vía de exclusión, se considera socialmente como una vía de integración, para ellos pierde su consideración de problema prioritario.

En segundo lugar, están quienes entienden este fenómeno como ausencia de valoración de la infancia. Esta perspectiva aunque en un principio suele ser adoptada también por los anteriores, sólo la mantienen a lo largo de todo su discurso aquellos que no tienen un contacto directo con el problema. Ello hace que su discurso sea menos emocional y apegado a lo concreto que el de sus compañeros, no discriminando entre las diferentes formas de TIJ , puesto que todas atentan contra los derechos del niño.

El discurso de los sindicalistas muestra, de nuevo, diferencias con los anteriores. Éstos, por lo general, interpretan el fenómeno del TIJ poniéndolo en relación con el subempleo, la economía sumergida y la sociedad de consumo. Centran su discurso, principalmente, en aquellas tipologías de TIJ que están más relacionadas con la producción en sentido tradicional, dando un papel residual a aquellas otras vinculadas con la reproducción o con la producción en un sentido amplio. Desde esta perspectiva, el TIJ no es sólo execrable por interferir en el normal desarrollo del menor, sino por ser un síntoma de la perversión del sistema económico vigente y del retraso del proceso de modernización económica y social.

Por otra parte, si se analiza la forma en que abordan el fenómeno del TIJ 
aquellos informantes pertenecientes asociaciones u ONG también se detectan elementos diferenciales. Concretamente, se distinguen en este grupo dos tipos de discurso, de nuevo relacionados con si los informantes tienen, o no, conocimiento directo de la problemática.

En el primero, están los entrevistados que centran su discurso en aquellas formas de TIJ directamente relacionadas con la pobreza y la marginación. Éstos adoptan un concepto de TIJ amplio que da cabida a todas aquellas actividades que, por lo general, no son consideradas como trabajo en el sentido tradicional. Esto es, por una parte, todas aquellas actividades de menores que podrían englobarse en conceptos como buscarse la vida o trapichear e, incluso, algunas actividades delictivas. Y, por otra, en aquellas otras que podrían incluirse dentro del amplio concepto de ayuda familiar. Éstas últimas serían, bien de carácter reproductivo, bien de carácter productivo.

Este último conjunto de informantes no suele conceder mucha importancia en su discurso a otros tipos de TIJ. De hecho consideran que si en el entorno en el que trabajan se dieran casos de TIJ en la industria o los servicios, sería un mal menor, puesto que lo normal en estos ámbitos es la marginalidad y la ausencia de trabajo en sentido clásico.

«... Si un chaval de un barrio como estos de los que hablamos comienza a trabajar con 14 años en una platería, no es que lo veamos bien, pero la verdad es que a lo mejor tiene la oportunidad de acabar teniendo una vida normalizada en lugar de acabar en la calle o en el trullo».

El segundo tipo de discurso es el que mantienen los informantes no tienen un contacto directo con esta realidad. Para ellos el TIJ es una de las consecuencias de la ausencia de valores de una parte de la sociedad, o más bien, de la centralidad en su escala de valores de algunos que suelen tachar de erróneos, como el del consumismo.

En suma, del análisis realizado del discurso de los agentes sociales entrevistados sobre el fenómeno del TIJ se pueden extraer al menos tres conclusiones relevantes para esta investigación.

Primera, para todos los informantes el TIJ en general es un problema social muy importante desde un punto de vista cualitativo, esto es, con independencia de la cantidad de población afectada por el mismo.

Segunda, aunque a nivel teórico la mayoría de los entrevistados adoptan un concepto de TIJ que coincide plenamente con el se fija en la legislación 
vigente, buena parte de los mismos, especialmente los que tienen un contacto directo con el fenómeno, lo reinterpretan, explícita o implícitamente, cuando lo utilizan para analizar la realidad social que les rodea, adoptando entonces conceptuaciones que distan de la anterior.

Tercera, no todos tienen como referente el mismo tipo o tipos de TIJ cuando desarrollan su discurso. Por lo que cuando lo valoran como un problema importante no se están refiriendo a la misma realidad social.

\section{EL TRABAJ O INFANTO-J UVENIL EN ANDALUCÍA: TIPOLOGÍA, CAUSAS Y CONSECUENCIAS}

Si algo se ha puesto de manifiesto con claridad en el análisis de la percepción social del TIJ en Andalucía es que se trata de una realidad compleja o poliédrica. Por ello, toda pretensión de aprehenderla al completo es inviable y lo esencial de la misma sólo se puede establecer por referencia a valores o esferas valorativas. De esta forma se podrá resaltar una parte de la realidad y que ésta posea unidad, sentido y relevancia cognoscitiva.

Este convencimiento analítico nos alienta a construir una serie de tipos ideales ${ }^{5}$ de TIJ cuyo objetivo es ordenar intelectivamente la realidad estudiada. Esto es, que servirán para establecer las fronteras cualitativas que separan las diferentes formas de TIJ que existen an Andalucía y, por ende, para facilitar su comprensión y explicación.

En este artículo se han construido tres tipos ideales de TIJ en Andalucía que, en conjunto, tratan de seleccionar lo esencial de la realidad estudiada en relación con los objetivos de la investigación. Tales tipos ideales son: el trabajo por cuenta ajena, la ayuda familiar y, por último, la mendicidad y "el trapicheo". A cada uno de ellos se les ha dedicado un apartado, en el que se

5 Las características básicas de los tipos ideales en el sentido que aquí se utilizan son: (a) Son construcciones en si coherentes y unívocas referidas a la realidad, pero no son representaciones u homomorfismos de la misma. (b) Su construcción no se realiza por acotación extensiva de la realidad al modo de las definiciones clásicas sino que se refieren a ella de modo ostensivo. (c) Son susceptibles de cierta generalización pero no tienen carácter nomológico. (d) Se construyen por exageración parcial de algún(os) aspecto(s) de la realidad, según categorías subjetivas pero no arbitrarias. (e) Su función es crear contextos de sentido, para a partir de ahí guiar la atribución causal y contribuir a la construcción de contextos causales probables 0 , al menos, plausibles.

Rodríguez Martínez, J., tipo ideal, voz en Giner, S., Lamo de Espinosa, E. y Torres, C. (1998) Diccionario de Sociología. Madrid, Alianza Editorial 
definen atendiendo a sus principales características; se ejemplifica y localiza en la realidad andaluza actual, y, por último, se trata de profundizar en las causas y consecuencias que tiene, en sí mismo, y para los diferentes agentes sociales atendidos en la investigación.

Con ello se pretende profundizar en el conocimiento del TIJ de forma integrada, es decir, tratando a la vez su fenomenología -su expresión en la realidad-, su casuística -aplicación de los principios morales a los casos concretos- y su conceptualización social.

\subsection{EI TIJ por cuenta ajena}

Este tipo ideal hace referencia a aquellas formas de TIJ que forman parte del concepto clásico de trabajo - trabajo asalariado-, esto es, incluirá aquel trabajo realizado por un menor de 16 años bajo la dirección y organización de otra persona a cambio de un salario. No se incluirá en esta tipología a aquellos menores que trabajen en el negocio o empresa familiar, excepto que tal actividad pueda ser claramente diferenciada de lo que posteriormente definimos como ayuda familiar.

Generalmente, el TIj que se incluye en este tipo ideal se realiza en empresas pequeñas o medianas en las que buena parte de su actividad se podría incluir en la llamada economía sumergida. No es habitual actualmente que se de este tipo de TIJ en las grandes empresas, ni en aquellas, sean del tamaño quesean, en las quelos sindicatos tienen presencia. Tampoco resulta habitual encontrar trabajadores de menos de 14 años realizando este tipo de TIJ , por ello, hasta el momento en que se amplió la escolarización obligatoria hasta los 16 años, no interfería de forma directa con la escolarización de los menores. En este sentido, no obstante, actualmente podemos encontrar dos tipos de situaciones diferentes. Por un lado, aquella en la que se encuentran los menores que trabajan por cuenta ajena durante todo el año. Y, por otro, aquella en la que están los menores que son trabajadores por cuenta ajena solamente durante el período estival, vacacional o en los días u horas en los que no tienen obligación de ir a la escuela.

El primero de estos grupos estaría formado por menores que no están escolarizados o que lo están pero no asisten. Mientras que, en el segundo grupo, la actividad laboral de los menores no impide su escolarización, aunque sí suele interferir en ella provocando, según los educadores, mayor fracaso escolar. 
Otra característica importante es que, por lo general, los menores que pertenecen al primer grupo, suelen trabajar en empresas del sector industrial. Mientras que los que realizan su actividad laboral en los distintos períodos vacacionales suelen trabajar en el sector de los servicios y, más en concreto, en la hostelería. No obstante, en el sector productivo agrario se pueden encontrar ambos tipos de situaciones.

Y la última característica destacable de los menores que se pueden incluir dentro de este tipo ideal es que, por lo general, no pertenecen a familias excluidas, sino más bien, a familias normalizadas de clase obrera aunque con un bajo nivel cultural.

\subsubsection{Principales causas y consecuencias del TIJ por cuenta ajena en Andalucía}

Lo primero que podemos afirmar sobre el TIJ por cuenta ajena, tal y como lo hemos caracterizado anteriormente, es que, por lo general, la sociedad andaluza es bastante permisiva con él. De hecho, en muchas ocasiones, éste no se considera como una forma de explotación o exclusión social del menor, sino que suele ser valorado como una vía de integración social de aquellos jóvenes que, por diferentes motivos, de no estar trabajando estarían expuestos a situaciones de riesgo (drogas, delincuencia,...). El miedo que subyace a este razonamiento empuja a los propios familiares a incentivar al menor para que trabaje, e incluso, a buscarle dicho trabajo entre conocidos y amigos.

Sin embargo, por lo general, no hay una sola causa o un solo factor que explique el fenómeno; más bien, la explicación del mismo reside en un conjunto de factores que, en muchos casos, se dan conjuntamente.

El primero es el fracaso escolar. En la mayoría de los casos los menores que acaban realizando un trabajo de estas características presentan un alto grado de fracaso escolar, lo cual, suele derivar en altas tasas de absentismo, en una cierta conflictividad escolar y familiar, y en el consiguiente abandono, formal o informal, del sistema educativo. Posteriormente, en algunos casos, su propia familia puede animarle a trabajar para preservarlo de ambientes socialmente insalubres o para demostrarle que si no sigue estudiando eso es lo que le espera.

En estos casos, a nuestro juicio, el trabajo por cuenta ajena no es la causa del abandono del sistema educativo, sino que se trata de una de sus 
consecuencias. No obstante, en algunos casos, pocos, la relación puede ser inversa, es decir, el abandono se produce como consecuencia de la facilidad que tiene el menor de incorporarse al mundo del trabajo y de las ventajas que encuentra, económica y socialmente, en hacerlo. Ello es lo que suele ocurrir en los lugares que podemos denominar como nuevos focos de TIJ $^{6}$, en los que resulta normal que el razonamiento de familiares y menores se dirija al beneficio económico inmediato, frente al educativo.

Otro factor explicativo del TIJ por cuenta ajena es la consideración del trabajo como un agente socializador positivo para los jóvenes. Este tipo de razonamiento aunque no es exclusivo de ningún estrato social concreto, parece más frecuente entre las familias obreras que tienen un nivel de formación bajo y, sobre todo, en aquellas en que alguno de sus progenitores trabajó durante su infancia.

No obstante, este factor explicativo no suele ser ni el único, ni el principal, cuando el TIJ por cuenta ajena se convierte en la actividad principal del menor, aunque sí es muy importante cuando el trabajo lo realiza el menor exclusivamente durante sus vacaciones escolares o los fines de semana.

Destaca en el análisis del TIJ por cuenta ajena otro factor directamente relacionado con el menor: la independencia económica y las posibilidades de consumo que proporciona el trabajo. Este factor es especialmente potente cuando el menor pertenece a una familia que, si bien no tiene por qué ser pobre, tiene una escasa capacidad de consumo de determinados productos:

6 Expresión que hace referencia a una serie de localidades que, comparten la característica de haber tenido un gran desarrollo económico en los últimos 10 ó 15 años. Los ejemplos más claros de las mismas serían Lepe (Huelva) o El Ejido (Almería). En estas localidades en las que la renta media per cápita es muy superior a la media andaluza y en las que el desempleo no es considerado como un problema, es en donde se han detectado con mayor frecuencia situaciones de TIJ , especialmente, a partir de los 14 años. Además, en todas ellas se detecta cierto cierre social en torno a este fenómeno, entre otros motivos porque, por lo general, no se considera como un problema sino, más bien, como todo lo contrario.

Las causas del TIJ no son la pobreza, la explotación de los menores, la falta de medios o recursos para estudiar, sino que, por lo general, se circunscriben, por ejemplo, a las posibilidades de consumo que les permite el trabajo a estos menores o la escasa valoración social de la educación y la formación. Es pauta común que muchos menores (del 20 al 25\% en Lepe) abandonen la educación obligatoria con 13 ó 14 años y comiencen a trabajar en el negocio de algún familiar o amigo. Como ya se ha dicho, este tipo de situaciones no crean alarma social en dichas comunidades por lo que, exteriormente, no se tiene demasiada información sobre el fenómeno, e internamente no se utilizan las vías legales o administrativas para paliarlo. 
motocicletas, ropa de marca, etc. También parece actuar de forma principal cuando se analizan situaciones de TIJ por cuenta ajena esporádicas y, de forma coadyuvante, cuando este tipo de trabajo no se realiza de forma esporádica sino que es la actividad principal del menor.

$Y$, el último factor explicativo que se ha identificado con cierta claridad en el análisis de este tipo de TIJ es el del reconocimiento social que obtiene el menor a través del trabajo, ya venga éste de la familia, de su grupo de pares, o de ambos. Dicho fenómeno está muy relacionado con la angustia que puede generar entre los menores el período de transición de la adolescencia. Es común que el adolescente que se incorpora al mundo del trabajo note cómo adquiere un estatus familiar, económico y, por ende, social que, de no trabajar, tardaría más tiempo en alcanzar. Ello es frecuente en aquellos ambientes sociales y/o familiares donde el estatus de un individuo está directamente relacionado con el dinero que es capaz de generar.

Si atendemos ahora a las consecuencias de este tipo de trabajo en la realidad andaluza actual, tenemos que reconocer que los informantes, por lo general, fueron mucho menos prolijos que al hablar sobre su incidencia o sobre sus causas, lo cual en principio puede ser entendido como un indicador de la permisividad social ante este tipo de TIJ de la que se habló anteriormente.

No obstante, aquellos que se pronunciaron lo hicieron principalmente en torno a tres tipos de consecuencias. La primera pone en relación el TIJ con el desarrollo en los menores de actitudes o pautas de consumo impropias de su edad. Éstas abarcan desde el consumo de tabaco, alcohol y otras drogas, hasta la habitualidad de embarazos no deseados y matrimonios que, por la inmadurez de los cónyuges, pueden derivar en situaciones de malos tratos, abandono u otras de riesgo.

La segunda, la citan aquéllos que afirman que el TIJ crea en el menor falsas expectativas con respecto a su futuro laboral. Éstos consideran que actualmente la especialización y la formación son elementos fundamentales para obtener un trabajo normalizado. La falta de tal especialización, influirá negativamente a aquellos que deseen normalizar su situación laboral, ya que encontrarán mayores dificultades, estando más expuestos al paro, la desprotección laboral y a los bajos sueldos.

$Y$ el tercer y último tipo de consecuencias agrupa a los que consideran que este fenómeno tenga o no consecuencias directas para los afectados, las 
tiene para la sociedad en general. En el corto plazo, porque potencia la economía sumergida, la competencia desleal y frena la creación de puestos de trabajo normalizados. Y, en el medio y largo plazo, porque descapitaliza y polariza la sociedad.

\subsubsection{Sobre la gravedad del TIJ por cuenta ajena}

El discurso de los diferentes agentes sociales entrevistados sobre la gravedad de este tipo de TIJ es distinto. De hecho, si estableciéramos un continuo en el que en un extremo estuvieran aquellos que lo consideran un grave problema social y en el otro, los que le otorgan menos importancia, colocaríamos en el primer extremo a educadores y sindicalistas y, en el segundo, a los jueces de menores y a los entrevistados de los servicios sociales de barriadas marginales.

No obstante, es interesante destacar que aquellos que consideran este tipo de TIJ como un grave problema no parecen considerarlo así por las consecuencias directas que éste pudiera ocasionar a los menores que lo realizan sino, más bien, por las implicaciones económicas, culturales o, en general, sociales que el mismo acarrea. Por ejemplo, los sindicalistas piensan que este tipo de TIJ es más preocupante por el hecho de fomentar la economía sumergida, el subempleo o la competencia desleal, que por creer que este tipo de trabajo, en general, puede tener graves consecuencias físicas, psíquicas o de otro tipo en los jóvenes que lo realizan - se refieren al tipo de menores que por lo general hacen este trabajo, es decir de entre 14 y 16 años.

Sin embargo aquellos otros que consideran que el TIJ por cuenta ajena es un fenómeno preocupante, pero no de extrema gravedad, centran su discurso en los beneficios que puede tener este tipo de trabajo sobre menores que se encuentran en situaciones de riesgo social. Ello no quiere decir que los jueces de menores o los trabajadores sociales de barriadas marginales no consideren denigrante el TIJ, sino que entienden que, en muchos de los casos que acostumbran a tratar, este tipo de TIJ puede ser un mal menor e incluso una vía de integración.

Todo ello invita a considerar que los agentes sociales cuando valoran la gravedad de este tipo de TIJ lo hacen desde criterios morales más que jurídicos, aunque siguen considerando estos últimos como el ideal a alcanzar. 


\subsection{La ayuda familiar como forma de TIJ}

El segundo tipo ideal de TIJ que parece relevante es el denominado Ayuda Familiar. Se trata de un concepto muy amplio que, en lo referente a los menores, puede abarcar desde situaciones que comúnmente no se consideran como trabajo, a otras, que por sus características, son más cercanas a este último concepto que al de ayuda. Para discernir entre unas y otras y centrar nuestro análisis en las segundas, se comenzará caracterizando este tipo ideal de TIJ .

La ayuda familiar de los menores puede tener un doble objetivo. Por un lado, ayuda familiar en labores reproductivas y, por otro, ayuda familiar en labores productivas. Ambas pueden considerarse en distintos casos como una forma de TIJ .

En el primer caso, la ayuda familiar se considera en esta investigación como una forma de TIJ siempre que impida la asistencia normalizada de los menores a la escuela. También, cuando, a pesar de no impedirla, el menor sea el último responsable en la realización de dichas tareas. Y, por último, cuando el tiempo que el menor tiene que dedicar a la realización de las mismas le impida habitualmente realizar otras actividades propias de su edad como, por ejemplo, jugar o relacionarse con su grupo de pares.

En el segundo de los casos, es decir, cuando el menor ayuda a su familia en labores productivas, se considerará como TIJ, además de en los tres casos anteriores, cuando dicha ayuda se preste de forma continuada; cuando el cumplimiento de la tarea del menor no esté supervisada por un familiar adulto; y, por último, cuando tal ayuda pueda menoscabar su salud física o psíquica y/o exponerlo a ambientes de riesgo.

No obstante, aunque esta caracterización establece los límites de las actividades de ayuda familiar que a priori se consideraron como TIJ en esta investigación, la aplicación del sentido común es, en la mayoría de los casos, más que suficiente para discernir entre las situaciones que son propias de la ayuda familiar y las que no lo son, es decir, aquellas que se deben incluir dentro de este tipo ideal de TIJ ${ }^{7}$.

7 Por ejemplo, por el lado de la ayuda familiar reproductiva no se considera como integrante de este tipo ideal de TIJ el que un menor que esté obligado por su familia a desarrollar ciertas tareas en casa como hacer su cama, recoger su habitación o fregar los platos. Sin embargo, si tal menor es el responsable de sus hermanos más pequeños de forma habitual, tanto para levantarlos, como para alimentarlos u otras labores de esta naturaleza, independientemente 
Resulta interesante destacar que en la ayuda familiar como forma de TIJ no se puede establecer, en Andalucía, una edad tipo en la que comience el fenómeno. Sin embargo, en las labores productivas, este tipo de TIJ aparece con mayor frecuencia conforme más edad tiene el menor. No obstante, en determinados ámbitos culturales, más que la edad, lo que marca el momento en que un o una menor debe de comenzar a realizar un trabajo de estas características es la concepción que la familia tiene de cuando el/la menor deja de serlo. Es habitual, por ejemplo, que en la cultura gitana este momento comience, para las niñas, con su primera menstruación y, para los niños, cuándo su desarrollo muscular parezca justificarlo.

Resulta interesante, no obstante, subrayar que la variable género si bien no es muy discriminante en cuanto a la frecuencia con la que se puede dar este tipo de TIJ , sí lo es en cuanto a la actividad que desarrollan los menores. Concretamente, en este ámbito se reproducen si cabe con mayor fuerza que en la sociedad andaluza en general, la asignación de papeles sociales en razón al genero. Siendo así que las labores reproductivas las realizan generalmente las menores y las productivas los menores.

$Y$, por último, se puede destacar que mientras que este tipo de TIJ se encuentra en sus dos vertientes, productiva y reproductiva, en el hábitat rural, en el urbano es más frecuente que se de en su vertiente reproductiva en los ámbitos de exclusión y entre las familias de clase obrera poco cualificada, y en la productiva, entre las clases medias patrimoniales -comerciantes y pequeños propietarios de hostelería.

4.2.1. Localización y frecuencia de la ayuda familiar como tipo ideal de trabajo infanto-juvenil

Por la información recabada este es el tipo de TIJ que se produce con mayor frecuencia en la sociedad andaluza actual. Además, en sus diferentes formas, se extiende por toda la comunidad autónoma. Por ello, en este

\footnotetext{
de que sea o no absentista, debe de ser considerado como un menor que trabaja, formando parte de este tipo ideal de TIJ.

Por el lado de la ayuda familiar productiva, no se considera como integrante de este tipo ideal de TIJ a aquel menor cuya familia tenga, por ejemplo, una tienda y que, en alguna ocasión, ayude a reponer las estanterías o a otras labores por el estilo. Sin embargo, sí se le consideraría como trabajador, si esta es una tarea que tiene que hacer obligatoriamente y de forma habitual, ya sea en sus horas de asistencia a la escuela o en las de ocio.
}

\section{RFS}


apartado trataremos de localizarlo con criterios diferentes a los geográficos. De hecho, la ocupación del o de la cabeza de familia y la clase social, en sentido amplio, parecen las variables que discriminan más en este sentido.

Desde este punto de vista, el primer foco importante de ayuda familiar como TIJ se da en Andalucía entre las clases medias patrimoniales, tanto urbanas como rurales. Concretamente, los pequeños agricultores, los comerciantes y los pequeños propietarios de la hostelería son los que con mayor frecuencia traspasan los límites anteriormente establecidos de la ayuda familiar como forma de TIJ . No obstante, dentro de este amplio grupo, el TIJ no se da siempre con la misma frecuencia: ésta parece fluctuar de forma inversamente proporcional al nivel de formación de los progenitores.

EI TIJ que suele detectarse en este grupo es el que socialmente está menos penalizado por la sociedad, entre otros motivos, por considerar que aunque en ocasiones discrimina al menor en cuanto a sus posibilidades de formación, en general, posibilita su integración laboral futura en el ámbito del negocio familiar.

El segundo grupo en el que se observa con frecuencia este tipo de trabajo es el que formarían aquellas familias sustentadas por trabajadores no asalariados de baja cualificación y formación, o artesanos que por su nivel derenta podríamos incluir en la clase obrera. Entre estos los más destacados son los jornaleros, los chapuzas y aquellos artesanos que trabajan de forma autónoma en su domicilio familiar y/o en ambitos de economía sumergida.

En este grupo, la ayuda familiar como forma de TIJ suele ser consecuencia del fracaso escolar y no causa del mismo. En él podemos encontrar menores con características similares a los que encontrábamos en el TIJ por cuenta ajena. De hecho, la principal diferencia es que en lugar de que la familia propicie a través de sus contactos la integración laboral del menor en una empresa ajena, por la propia idiosincrasia del trabajo que realizan o por la falta de dichos contactos, lo introducen en la actividad que ellos mismos realizan para ganarse la vida.

Y, el tercer y último foco de este tipo de TIJ lo encontraríamos entre aquellas familias socialmente excluidas y generalmente pobres en las que la ausencia de hecho de los progenitores, o la dejación de sus funciones por problemas como la drogadicción u otros, hacen preciso el trabajo de los menores para el sostenimiento familiar en términos productivos y/o reproductivos. 
La ayuda familiar que se produce en este último grupo es la que estaría peor considerada por la sociedad andaluza actual y, por ende, la que más unánimemente se considera como un problema social grave digno de erradicación.

En los dos primeros grupos, este tipo de TIJ puede encontrarse como actividad principal del menor y como actividad complementaria que no impide su escolarización obligatoria, siendo la segunda modalidad la más frecuente. No obstante, en el tercero de los grupos, la ayuda familiar se convierte en su actividad principal desde edades tempranas. Ello, además de impedir la escolarización, generalmente impide su futura integración social y laboral. De hecho, la mayoría de estos menores cuando llegan a la adolescencia acaban integrándose en ambientes delictivos.

4.2.2. Principales causas y consecuencias de la ayuda familiar como forma de trabajo infanto-juvenil en Andalucía

Entre los principales factores generadores de este tipo de TIJ se destacan los siguientes. En primer lugar, las diferentes conceptualizaciones culturales que perviven en Andalucía sobre cuando un niño deja de serlo. En segundo lugar, diferencias sobre cuál es el papel que debe cumplir en el seno de la familia cada uno de sus miembros. Y, en tercer y último lugar, diferencias sobre qué elementos son los óptimos en la socialización y educación de los menores.

Con respecto al primer factor es interesante comprobar cómo en la sociedad andaluza conviven diferentes formas de conceptualizar la llegada de la madurez, a menudo muy diferentes al concepto normativo-legal. Este fenómeno parece estar en la base de que, en determinadas familias, la ayuda de los menores traspase los límites antes establecidos y se convierta en una forma de trabajo. En estos casos, la causa del trabajo de los menores no sólo hay que buscarla en el beneficio económico, o de otro tipo, que aportan a sus familias, sino más bien en la preparación para la vida adulta que sus familiares pretenden conseguir obligándolos a realizar estas cuando consideran que han dejado de ser niños. Además, en muchos casos, consideran que su forma de establecer cuando llega este momento está naturalmente justificada puesto que se basa en manifestaciones biológicas (primera menstruación, desarrollo muscular, etc.).

Este factor explicativo del TIJ como ayuda familiar, en algunas ocasiones, 
está relacionado con las diferentes concepciones que tiene la población andaluza sobre cuál es el papel que cada miembro de la familia debe de cumplir. Este fenómeno ha estado tradicionalmente vinculado en Andalucía a las economías rurales de subsistencia, sin embargo, en cierto modo más o menos suavizado, ha calado en la cultura y formas de vida de algunos sectores de la población andaluza actual, dando lugar o justificando la ayuda familiar como forma de TIJ.

Y, por último, el tercer factor explicativo de este tipo de TIJ, es la concepción que tienen ciertas familias andaluzas de que el trabajo es parte integrante, cuando no fundamental, del proceso de socialización o formación del menor. El ejemplo más claro lo encontramos entre las clases medias patrimoniales que, en algunos casos, incentivan esta forma de TIJ con objeto de dotar al menor de las aptitudes y conocimientos necesarios para que en el futuro se pueda hacer cargo del negocio familiar. En los casos en que este último es el factor que mejor explica que un menor realice actividades que podemos considerar como TIJ , el menor, por lo general, sigue integrado en el sistema educativo, es decir, la ayuda familiar se compatibiliza con la escolarización obligatoria. Sin embargo, cuando este factor no es el principal, sino que se presenta junto con alguno de los otros dos mencionados, el trabajo suele impedir la escolarización del menor, lo cual sucede generalmente a partir de los 13 ó 14 años.

No obstante, además de estos factores principales para la explicación del fenómeno, es necesario destacar otros condicionantes que se presentan con cierta frecuencia en algunos de los casos que incluimos en este tipo ideal. Los más importantes son, las necesidades económicas y ciertas formas de desestructuración familiar. El primero de ellos es habitual en familias que por su precaria situación económica no pueden mantener ocioso a ninguno de sus miembros útiles para el trabajo. El segundo, menos evidente, se produce en aquellas situaciones de exclusión en las que se da una ausencia total o parcial de los progenitores, teniendo necesariamente los menores que asumir papeles productivos o reproductivos que no le son propios.

Estos condicionantes no se han considerado como factores principales para la explicación de este tipo ideal de TIJ porque en general y por si solos explican pocos de los casos que se pueden incluir en el mismo.

Por el lado de las consecuencias de la ayuda familiar como forma de TIJ es interesante subrayar que cuando el mismo no interfiere, al menos visiblemente, la escolarización del menor no suele producir alarma social y los 
informantes destacan del mismo principalmente sus consecuencias positivas, obviando o quitando importancia a las negativas. Por contra, cuando dicha situación obstaculiza visiblemente la escolarización del menor se resaltan más las consecuencias negativas del fenómeno que las positivas, considerándose entonces como un grave problema social.

Ello es interesante desde el punto de vista del cambio social porque refleja que la educación obligatoria actualmente tiene una alta consideración entre la sociedad andaluza, lo cual es síntoma del desarrollo cultural de esta comunidad. Sin embargo, también lo es desde un punto de vista analítico porque sorprende comprobar que la ayuda familiar como forma de TIJ no se valora mejor o peor, o crea más o menos alarma social, en función de las necesidades que la puedan generar, o del conjunto de consecuencias objetivas que pueda tener para el menor, sino en relación con una sola: si el menor asiste o no a la escuela, lo cual, además, ayuda a comprender por qué cuando éste sigue asistiendo regularmente a la escuela pese a trabajar, socialmente no está ni mal visto, ni penalizado.

Otra consecuencia deestetipo deTIJ que explícita o implícitamentesuelen reconocer la mayoría de los informantes, es la reproducción del fenómeno y de otras pautas discriminatorias asociadas con él. Es decir, una persona que ha realizado este tipo de trabajo en su familia de origen, tiende a considerarlo como algo normal y, cuando crea su propia familia, por lo general, trata de reproducirlo. Este fenómeno no era tan evidente, por ejemplo, en el TIJ por cuenta ajena. Además,en los citados núcleos familiares, con frecuencia, se afianzan y reproducen también pautas de conducta, por ejemplo, relacionadas con el género, que en otros ámbitos de la sociedad andaluza comienzan lentamente a superarse. Ello se observa con cierta claridad cuando comprobamos que el tipo de ayuda familiar que generalmente prestan las menores está vinculada a las labores de reproducción, frente a la que prestan los menores más orientada a las labores de producción.

La explicación de dicho fenómeno reproductivo podría encontrarse en que los menores que realizan este tipo de trabajo como actividad principal se socializan casi exclusivamente en el seno de su familia, interviniendo de forma poco significativa otros agentes socializadores como la escuela, lo cual repercute en que los valores y actitudes que adquieren sean, con mayor probabilidad que en otros casos, similares a los de sus padres. 
4.2.3. Sobre la gravedad de la ayuda familiar como forma de trabajo infantojuvenil en Andalucía

La valoración que hacen los diferentes agentes sociales de esta forma de TIJ es tan heterogénea como la propia realidad a la que se refieren. No obstante, en términos generales, se puede afirmar que este es el tipo de TIJ que menos alarma social produce, excepto en aquellos casos en los que está propiciado por fenómenos evidentes de exclusión social o que atañe a niños menores de 12 años.

Además, la mayoría de los entrevistados contemplan con mucho respeto el fenómeno al producirse en el ámbito privado de la familia de ahí que pongan mucho cuidado tanto en el discurso que construyen en torno él, como en la valoración que hacen del mismo.

Junto a ello es interesante comprobar, por una parte, que si la ayuda familiar no tiene contraprestación económica alguna se considera menos grave que si la tiene, lo cual, a nuestro juicio, incrementa aún más las situaciones de explotación y discriminación que se producen. Y, por otra, que si las actividades que realiza el o la menor son de carácter reproductivo son menos consideradas como trabajo y, por tanto, menos graves, que si son de carácter productivo. Por tanto, se puede afirmar que la ayuda familiar como forma de TIJ , socialmente no siempre se considera en Andalucía como tal, aún menos si no está remunerada y, casi nunca, si es de carácter reproductivo.

En suma, este tipo de TIJ por producirse en el ámbito privado, por no considerarse socialmente siempre como tal y por no crear alarma social en la mayoría de los casos, puede ser el más difícil de erradicar, lo cual se retroalimenta con que jurídicamente, en España, no se comprende la ayuda familiar sin relación asalariada como una forma de trabajo, al entenderse inexistente el vínculo contractual.

\subsection{Otras formas de TIJ : la mendicidad y el "trapicheo"}

En este apartado se caracteriza y analiza el último tipo ideal de TIJ construido para la descripción y análisis de este fenómeno en Andalucía. En él se incluyen dos formas distintas de trabajo de menores puesto que, a pesar de ser diferentes en su casuística, son parecidas tanto en sus consecuencias como en el tipo de población en el que más frecuentemente se dan. 
Por un lado, tenemos el trabajo de menores en la mendicidad. En este sentido hay que distinguir entre aquellos menores que son utilizados para practicar la mendicidad y aquellos menores que la practican. En el primer caso se trata generalmente de niños de 0 a 3 años que son utilizados, habitualmente aunque no siempre, por algún familiar como reclamo en su labor mendicante. En el segundo caso se detectan dos situaciones diferentes. Por un lado, la de aquellos menores que mendigan de forma esporádica para obtener algo de dinero para sí mismos y, por otro, aquellos que lo hacen de forma habitual y más o menos organizada en el seno de un grupo que se dedica a esta labor. En ambos casos los menores que desarrollan esta actividad pertenecen a familias y barriadas con un alto nivel de exclusión y de pobreza; viven situaciones de abandono total o parcial; no han estado escolarizados nunca o han sido absentistas habituales en la primaria y crónicos en la secundaria; pertenecen a familias cuyos progenitores son por lo general muy jóvenes -en muchos casos tuvieron hijos cuando aún eran adolescentes- y tienen alguna toxicomanía y/o problema con la justicia.

No obstante, es importante destacar que en algunos casos es difícil deslindar este tipo de TIJ de la ayuda familiar. De hecho, podemos encontrar algunos casos en los que los niños practican la mendicidad como forma de trabajo, siguiendo la tradición familiar y bajo la supervisión, organización y disciplina del cabeza de familia. En estos casos, las características ambientales y familiares del menor son algo diferentes, puesto que no se suelen encontrar en situación de abandono, realizan dicha actividad al amparo de eventos 0 acontecimientos festivos y tienen una forma de vida prácticamente nómada.

Por otro lado, en este tipo ideal de TIJ se incluyen también menores que se dedican a trapichear o buscarse la vida. Estos tienen, por lo general, un ambiente familiar y social similar al anteriormente expuesto, sin embargo, el tipo de trabajo que realizan es diferente. Por lo general son menores que se crían en la calle y realizan esporádicamente algún tipo de actividad delictiva: hurtos a pequeña escala, correos para el contrabando de tabaco o drogas, venta de hachís, o vigilancia y alerta de la presencia de policía en la zona. En otras ocasiones, las menos, realizan actividades como la venta de periódicos, recogida de cartón o chatarra, o montaje y desmontaje de atracciones de feria, etc.

El trapicheo como forma de TIJ es más frecuente entre los menores que entre las menores que, por regla general en estos ambientes, están sometidas 
por los padres o hermanos y realizan labores de reproducción familiar.

La edad no es una variable que discrimine de forma relevante en este tipo de TIJ. De hecho, en la mendicidad son utilizados como reclamo cuando aún no se valen por sí mismos y la practican, de una forma u otra, cuando son mayores. Y, en el trapicheo, se pueden encontrar menores de todas las edades, aunque por lógica es más probable entre los menores que han alcanzado la edad de 11 ó 12 años.

El hábitat sí es una variable muy importante en la caracterización de esta población puesto que, en la mayoría de los casos, este es un fenómeno urbano. En concreto se produce en las barriadas chabolistas y de mayor exclusión de las grandes ciudades.

Desde un punto de vista étnico, parece que la mendicidad se produce más frecuentemente entre las familias gitanas excluidas y pobres y, en los últimos años, entre los gitanos rumanos asentados en nuestra comunidad. Mientras que, para las otras formas de TIJ incluidas en este tipo ideal, esta variable no es discriminante.

4.3.1. Localización y frecuencia de la mendicidad y el «trapicheo» como formas de trabajo infanto-juvenil en Andalucía.

Este tipo de TIJ , como se ha señalado anteriormente, es un fenómeno más frecuente en las áreas o barriadas marginales de las grandes ciudades andaluzas.

Con respecto a su frecuencia, creemos acertado decir que en términos relativos es el tipo de TIJ menos importante en Andalucía, aunque socialmente es el que más alarma produce y sobre el que más acuerdo hay en que es una forma de explotación infantil digna de ser totalmente erradicada.

No obstante, la información obtenida parece apuntar dos tendencias susceptibles deser mencionadas. Por una parte, es unánime la consideración de que la mendicidad como forma de TIJ ha descendido notablemente en Andalucía en la última década. Y, por otra, es también habitual que los entrevistados en contacto con las demás formas de TIJ que se incluyen en este tipo ideal, consideren que éstas, ponen a los menores en situaciones que cada vez son de mayor riesgo social, incrementando y reproduciendo las bolsas de exclusión y pobreza severa existentes en Andalucía.

Este último tipo de TIJ está frecuentemente relacionado con el tráfico de 
drogas y, en algunas ciudades concretas, también con el contrabando de tabaco. Lo cual se aprecia con especial claridad en los barrios marginales de Algeciras, La Línea de la Concepción y Málaga. Ambientes en los que los menores realizan labores de vigilancia o de transporte de drogas o tabaco de contrabando, a cambio de sustanciosos beneficios económicos que, a su vez, son empleados generalmente para el consumo de estas mismas sustancias 0 para la adquisición de productos que, por su situación familiar o por no ser propios de su edad, de otra forma tendrían vetados. Fenómeno que unido al descuido, abandono y falta de seguimiento de sus padres, provoca que estos menores además de ser analfabetos funcionales adquieran hábitos y conductas nocivas tanto para su salud física como psíquica.

Los menores que realizan este tipo de actividades no poseen un referente claro de lo que es un trabajo normalizado en su entorno social más cercano, llegando a despreciarlo de la misma forma que desprecian el estilo de vida que la sociedad les ha negado, adoptando actitudes violentas, antisociales y prepotentes con las que tratan de defenderse de su marginal situación.

En suma, por el tipo de información con la que contamos, no se puede establecer una jerarquía entre las distintas provincias andaluzas, que tenga como base la incidencia o frecuencia de estas formas de TIJ, sin embargo, parece oportuno subrayar que es un fenómeno que, en sus diferentes manifestaciones, es habitual entre la población más marginada de Andalucía.

4.3.2. Principales causas y consecuencias de la mendicidad y el «trapicheo» como tipo ideal de trabajo infanto-juvenil en Andalucía

El factor que parece explicar mejor las actividades que se incluyen dentro de este tipo ideal de TIJ, es el ambiente de exclusión social y el abandono familiar en los que se desarrolla el menor. Por ello se podría decir, sin demasiado temor a errar, que este fenómeno es connatural a aquellos menores de familias excluidas socialmente y que viven en ambientes de extrema marginalidad.

La causa de la exclusión del menor no se encuentra en la realización de este tipo de actividades sino que se trata de la principal consecuencia de nacer en una familia excluida. Por tanto, este tipo de situaciones, en la mayoría de los casos, no están motivadas ni por la decisión de los padres, ni por la de los propios menores, sino que son el resultado lógico de la supervivencia y adaptación al medio de los menores de determinados ambientes marginales.

\section{RFS}


Dicho en otras palabras, consideramos que este es un fenómeno de segunda generación, es decir, que es un efecto perverso de una situación que tiene sus causas más atrás y no directamente en la decisión de los agentes implicados.Sin embargo, las consecuencias del mismo sí atañen directamente a los menores afectados e indirectamente a la sociedad en general. Las más destacables son las siguientes:

En primer lugar, la exclusión de estos menores del sistema educativo, bien porque no han estado escolarizados nunca por la desidia o el abandono de sus padres, bien por la inadecuación existente entre el ambiente social, económico y familiar en el que viven y los requerimientos de un sistema educativo que no se adapta a sus necesidades y expectativas.

En segundo lugar, su integración en el submundo de la marginalidad adoptando estilos de vida en los que la delincuencia, las toxicomanías, la violencia, etc., son la norma más que la excepción, lo cual los convierte en firmes candidatos a entrar en la cárcel, a tener una vida insalubre y corta y, en general, a sobrevivir en el día a día sin más posibilidades de futuro.

$Y$, en tercer y último lugar, el convertirse sin mucho margen de maniobra en agentes mantenedores y reproductores de ámbitos o bolsas de extrema marginación, exclusión y, generalmente, pobreza. Lo cual no sólo es un problema para ellos, sino una constricción para el futuro de su prole.

4.3.3. Sobre la gravedad de la mendicidad y el «trapicheo» como formas de trabajo infanto-juvenil

Si bien sobre los otros dos tipo de TIJ construidos para el análisis del trabajo de los menores en Andalucía, se han encontrado discursos divergentes en cuanto a la importancia o gravedad del fenómeno, en el caso que se aborda en este último apartado la convergencia en el discurso es lo habitual. De hecho, todos los entrevistados parecen coincidir tanto en la gravedad social que tiene este fenómeno como en la ausencia de consecuencias positivas, aunque sean parciales, del mismo. Además, mientras que los entrevistados construían básicamente su discurso sobre los otros dos tipos de TIJ , sobre la base de los efectos positivos o negativos del mismo en el ámbito que más conocían, a saber, los sindicalistas en el ámbito del trabajo, los educadores en el de la formación y la educación etc., cuando lo hacían sobre este último, lo abordaban como un problema social global y difícilmente abordable sectorialmente 


\section{A MODO DE CONCLUSIÓN}

EI TI en Andalucía es un fenómeno social desconocido tanto cuantitativa como cualitativamente. Lo cual no es un problema exclusivo de esta Comunidad Autónoma sino del conjunto de los países occidentales desarrollados. Probablemente, la causa de dicho desconocimiento se encuentre en lo que UNICEF ha denominado el primer gran mito sobre el TIJ en el mundo, a saber, considerar que éste es un problema de los países pobres. No obstante, se ha comprobado que se trata de un problema general, de ahí que, en los últimos años, algunos países desarrollados se hayan interesado especialmente en él, lo estudien y traten de ponerle fin, en la medida de sus posibilidades.

Lo primero a tener en cuenta al afrontar el estudio del TIJ es que se trata de una realidad compleja. Es un fenómeno que adopta diferentes formas que tienen causas y consecuencias diferentes. Ello y la falta de indicadores que lo recojan, dificulta enormemente tanto su conocimiento como su medición. De hecho, aunque se encontraran tales indicadores, de no haberse cualificado previamente el fenómeno, no se sabría con seguridad qué se está midiendo realmente con ellos.

En cuanto a la cuantificación del fenómeno, pese a que éste no era uno de los objetivos principales de la investigación que aqui se presenta, se ha comprobado cómo la mayoría de los entrevistados consideraba que este fenómeno ha decrecido en los últimos años. Lo cual pudiera ser el reflejo de un cambio cualitativo y sectorial del TIJ del que sí se ha obtenido constatación empírica. Ejemplo de ello es la disminución de este tipo de trabajo en poblaciones y sectores productivos que socialmente se consideran como focos de TIJ , como es el caso de los jornaleros y el sector agrícola en general.

Sin embargo, también se han detectado una serie de focos de TIJ en los que este fenómeno, lejos de decrecer, está aumentando afectando en términos relativos a un contingente importante de menores. Estos focos, por lo general, se sitúan en ámbitos geográficos muy localizados en los que ha habido un importante y rápido desarrollo económico que no ha ido acompañado por un desarrollo cultural del mismo calibre, lo cual ha tenido como efecto perverso que muchos menores y sus familiares hayan considerado que el trabajo, que es un bien escaso en la sociedad andaluza en general, es la vía más segura y rápida para su integración social, despreciando o infravalorando la educación y la formación académica.

Por otra parte, es interesante resaltar que si bien la pobreza, la etnia o el 
sector productivo no han sido variables determinantes a la hora de analizar en general el TIJ en Andalucía, sí lo han sido la clase social, la exclusión, la formación de los padres y el género. De hecho, se ha comprobado cómo este fenómeno en sus diferentes tipologías es más habitual entre las familias de clase media patrimonial, las de obreros poco cualificados y con escasa formación, y las excluidas y marginadas por diferentes motivos. Pero, además, el tipo de trabajo que realizan los menores está fuertemente constreñido por el género de los mismos. Fenómeno que encuentra su explicación en la reproducción de valores tradicionales, en este caso machistas, que se produce en estas familias como resultado de la escasa presencia de agentes socializadores diferentes a ellas mismas.

Los dos factores que la sociedad andaluza actual considera más unánimemente como fundamentales para valorar si el trabajo que realiza un menor es digno de considerarse explotación o no, son la edad y la asistencia del menor a la escuela. Es decir, el TIJ ya sea por cuenta a ajena o como ayuda familiar, por lo general se considera malo o explotador cuando se produce en menores de 13 años y, especialmente, si éste impide la asistencia del menor a la escuela. Siendo así que si el trabajo no incumple ninguno de estos dos condicionantes, por lo general, no se valora como especialmente grave, siendo incluso susceptible de ser considerado como una vía de evitar problemas de mayor calad o como la anomia, la marginación o la delincuencia.

\section{BIBLIOGRAFÍA}

Barriga, S.; Ramírez, J .; J Iménez, M. y León, J. (1998), Mendicidad infantil. Una experiencia de intervención psicosocial en A. Martín González (ed), Psicología Comunitaria. Fundamentos y aplicaciones. Madrid, Síntesis Psicología, pp. 311-324.

Bequele, A. y Myers, W. (1995), Prioridad del trabajo infantil: la eliminación del trabajo perjudicial para los niños. Ginebra, Organización Internacional del Trabajo y UNICEF.

DNI - España y IWGCL (1995), El trabajo Infantil en España 1995. A Report to the International Working Group on Child Labour. Madrid.

IBÁÑEZ, J. (1994), Perspectivas de la Investigación Social: El diseño en las tres perspectivas en M. García Ferrando (coord.), El análisis de la realidad social. Métodos y Técnicas de investigación. Madrid, Alianza Universidad Textos. 
LieBeL, M. (1998), «iSí al trabajo - no a la explotación! Para un trato del trabajo infantil orientado hacia el sujeto», en A. Recknagel (ed), Arbeitende Kinder stärken. Plädoyers für einen subjektorientierten Umgang mit Kinderarbeit. Frankfurt/main, Verlag für Interkulturelle Kommunikation, 1998, pp.333357. Traducción: Francisco Mirallas y Dima Zito, Informationsbüro Nicaragua, Wuppertal.

MaRTínez, M. (1998), Repensar la mirada sobre la infancia trabajadora. Madrid. Comisiones Obreras.

OdPA (1997), Temporeros y Educación. La atención educativa a los hijos de trabajadores temporeros. Sevilla, Oficina del Defensor del Pueblo Andaluz.

ODPA (1998), El absentismo escolar. Un problema educativo y social. Sevilla, Oficina del Defensor del Pueblo Andaluz.

Oıт (1996a), ¡Alto! al trabajo infantil. Ginebra, Organización Internacional del Trabajo.

ОІт (1996b), El trabajo infantil: ¿Qué hacer? Ginebra, Organización Internacional del Trabajo.

Rojas FLORES, J. (1998), «El trabajo infantil en Chile: algunas ideas para el debate», en M. Fernández (ed), Economía y trabajo en Chile 1997-1998, Santiago, PET., pp. 129-194.

TAYLOR, S. y Bodgan, R. (1994), Introducción a los métodos cualitativos de investigación. La búsqueda de significados. Barcelona, Ediciones Paidós.

UGT (1992), Condiciones del trabajo de los niños en España. Madrid, Unión General de Trabajadores.

UGt (1996), Sin tiempo para jugar. El trabajo infantil en la economía mundial. Madrid, Unión General de Trabajadores.

UGT (1998), Stop al trabajo infantil. Madrid, Unión General de Trabajadores.

UnICEF (1997), Informe sobre el estado mundial de la infancia de 1997, Ginebra, UNICEF. 